\title{
Performance Evaluation of Multi-Interfaced Fast Handoff Scheme for PNEMO Environment
}

\author{
Shayla Islam ${ }^{1,3}$, Aisha-Hassan Abdalla ${ }^{1}$, Farah Nadia Mohd Isa ${ }^{1}$, Mohammad Kamrul Hasan ${ }^{2}$ \\ ${ }^{I}$ Department of Electrical and Computer Engineering, International Islamic University Malaysia, \\ Kuala Lumpur, Malaysia \\ ${ }^{2}$ Department of Electrical and Electronics Engineering, Universiti Malaysia Sarawak (UNIMAS), \\ 94300 Kota Samarahan, Sarawak, Malaysia \\ ${ }^{3}$ Department of Computer Science and Engineering, Green University of Bangladesh \\ hmkamrul@unimas.my
}

\begin{abstract}
Mobility management is classified into two parts such as location management and handoff management. The earlier one concentrates on location update whereas the later one manages continuous Internet connectivity while the Mobile Router (MR) changes its single point of attachment to the network. Therefore, frequent movement of the MR is one of the significant characteristics in Network Mobility (NEMO) environment. Because, in accordance with the standard Network Mobility Basic Support Protocol (NEMO BSP), the MR utilizes single Interface to attach to the access link. MR requires changing its Care of Address (CoA) when it moves among different wireless access networks. As a result, it can directly influence the performance of the mobility management protocols during inter technology handoff of multi-interfaced MR. This paper proposed a multi-interfaced fast handoff scheme in Proxy NEMO (PNEMO) environment. After that, it represents a comparative analysis between the proposed multiinterfaced scheme, NEMO BSP and the PNEMO scheme respectively. The performance disparities of these schemes are estimated and analyzed via both numerical and simulation approaches. The simulation is performed through NS-3 network simulator. The performance metrics estimated for evaluation are mainly handoff delay and packet loss. It has been perceived that, the proposed scheme performs better compared to the PNEMO scheme and NEMO BSP.
\end{abstract}

Index Terms-NEMO; NEMO BSP; PNEMO; Multiinterfaced MR; Mobility management.

\section{INTRODUCTION}

At the present time, mobility management with providing a continuous Internet connectivity is one of the ultimate demanding research concerns in Network Mobility (NEMO) environments. It becomes more difficult to meet all the prerequisite of NEMO by the conventional mobility management protocols with its extensions to support network layer mobility management [1]-[4]. This is due to the frequent movement of MR during handoff in NEMO. Therefore, the Network Mobility Basic Support (NEMO BSP) has been standardized to provide a continuous Internet

Manuscript received 30 November, 2017; accepted 12 July, 2018.

A special thanks to the Government of Malaysia, through Ministry of Education $(\mathrm{MoE})$ for the education sponsorship. This work is also partially supported by the RIGS16-351-0515, International Islamic University, Malaysia. connectivity of a cluster of Mobile Network Nodes (MNNs) by a MR in a mobile network [5], [6].

Since NEMO BSP is an amended version of MIPv6, it is still challenging issues for real time application scenarios due to increase handoff delay and packet loss during handoff. Therefore, in order to overcome these drawbacks of NEMO BSP, there have been some proposals linked to network-based schemes in NEMO manipulating the fast handoff feature on PMIPv6 in NEMO. Although, these schemes can provide better handoff performance, still required to survey the characteristics of each NEMO entities to provide seamless handoff during inter technology handoff (i.e. movement among different access networks). This is because; NEMO is concerned not only with the MR but also MNNs. Moreover, these schemes still experience a higher handoff delay due to extra tunnelling burden that leads to a lower throughput during inter technology handoff [7]-[17]. It is evident from existing evaluation results that, the handoff delay on Home Agent (HA) remains increasing with the increase number of MNNs. This leads to increase much more data traffic that is being routed via HA in NEMO [10][13]. Precisely, increment of the number of MNNs can enhance higher tunnelling burden and extra signalling overhead on the link between the present and new access routers.

The basis of this work is to know the functioning mechanism of the mobility management schemes and to determine which protocol provide better handoff performance. The contribution of this paper includes: (i) propose a multi-interfaced fast handoff scheme in PNEMO environment. (ii) develop a simulation scenario using NS 3 simulator to compare the applicability and efficiency of the proposed scheme with that of the PNEMO scheme and NEMO BSP (ii) Investigate and analyse numerical and simulation outcomes in terms of handoff delay, and packet loss.

The remaining portion of this paper is structured as follows: the proposed multi-interfaced fast handoff scheme is detailed in Section II. Then, performance evaluation is offered in Section III. Section IV present both numerical and simulation outcomes with analysis. Finally, the paper is concluded in Section V. 


\section{Proposed Multi-InTERfaced SCHEME}

The handoff occurs when the MR or MNNs moves between different wireless communications technologies. Multiple interface-based MR can attain multihoming features namely increased availability, balanced traffic load with flow distribution through simultaneous connectivity during inter-technology handoff. This is why delay during handoff is reduced [15]-[18].

With the aim of taking the benefit of using multiple interfaces, this paper proposes a multi-interfaced fast handoff scheme in PNEMO environment. According to the proposed scheme, the Serving MR (SMR) acts like an MR in PNEMO-scheme. In the proposed multi-interfaced fast handoff scheme, the Current MR (CMR) and the New MR (NMR) are used as a replacement of MAG1 and MAG 2 in PNEMO scheme. The CMR and NMR are liable for identifying the SMR's movements among different access networks. They are also responsible to differentiate and attain the Mobile Network Prefix (MNP) as well as Home Network Prefix (HNP) from an acknowledgment message which is directed from the local HA (i.e. LMA). Besides, CMR and NMR exchange the context of the SMR via L2 triggering mechanism. A reference analytical framework of the proposed multi-interfaced fast handoff scheme with the network entities is depicted in Fig. 1.

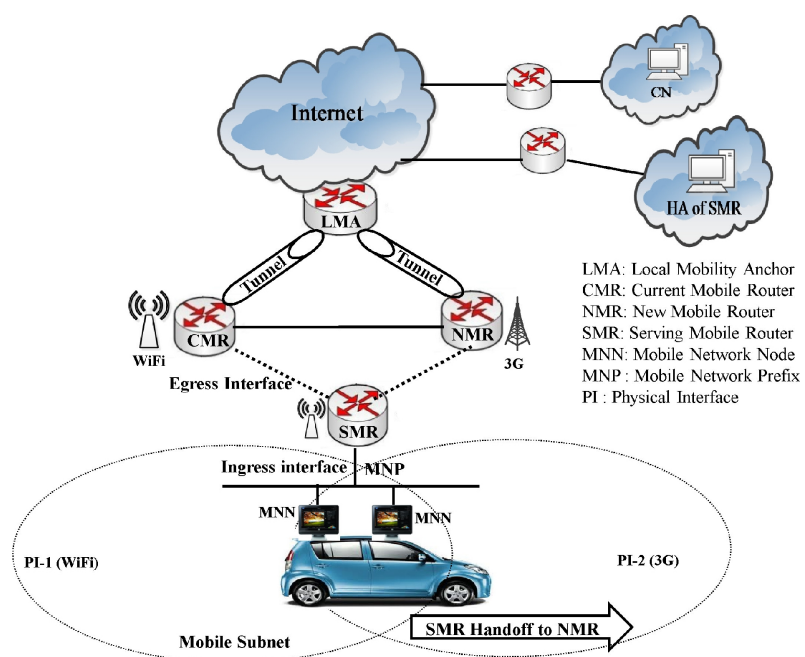

Fig. 1. Analytical framework of proposed multi-interfaced fast handoff scheme.

In the proposed scheme, the CMR and NMR exchange Handover Initiations (HI) and Handover Acknowledgement (HAck) messages earlier to the L2 handoff. This is to efficiently support seamless handoff in PNEMO environment. The HI message contains the accumulated information of the SMR such as the SMR ID, Home Network Prefix (HNP), Mobile Network Prefix (MNP), LMA address and so on. This accumulated information helps to enable the NMR to redirect a binding registration message with MNP option of SMR to LMA for accomplishing a location update process. In order to perform fast registration, an Early Proxy Binding Update (EPBU) and Early Proxy Binding Acknowledgement (EPBA) messages are encapsulated inside $\mathrm{HI}$ and Hack messages respectively. The proposed scheme is illustrated briefly using a flow diagram in Fig. 2.

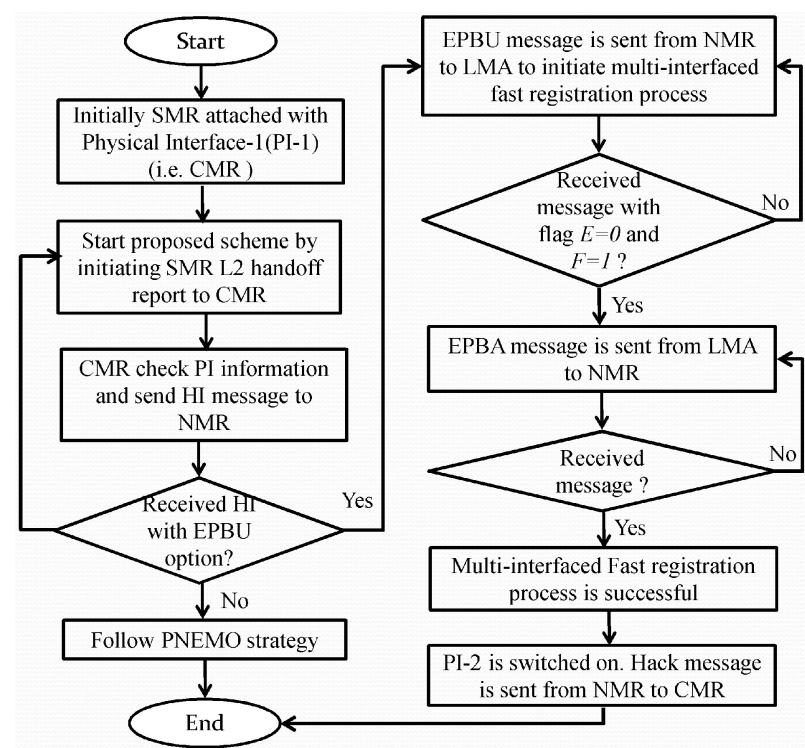

Fig. 2. Flow diagram of the proposed scheme.

\section{PERFormance EVAluation}

With the aim of evaluating the applicability and the efficiency of the proposed scheme, both numerical and simulation approaches are considered in this section.

\section{A. Numerical Evaluation}

The Handoff Delay (HD) of the SMR is identical to the totality of the times required to accomplish the functions such as Link Switching (LS), Movement Detection (MD), Care of Address (CoA) configuration as well as registration to notify the present location of the SMR. The Handoff Delay (HD) of the proposed scheme is calculated as

$$
T_{H D}^{\text {Multi-interfaced }}=T_{L S}+T_{S M F R}+T_{P R},
$$

where $T_{L S}$ is the delay of link switching, $T_{P R}$ is the Partial handoff delay of the proposed scheme required for postregistration, $T_{S M F R}$ is the Partial handoff delay of the proposed scheme required to support multi-interfaced fast registration. In (1), $T_{P R}$ and $T_{S M F R}$ are expressed as:

$$
\begin{aligned}
& T_{\text {SMFR }}= \\
& =\left[\begin{array}{l}
\mathrm{H}_{C M R-N M R} \times\left(\frac{L_{H I}}{B_{w d}}+t_{w d}\right)+\mathrm{H}_{C M R-N M R} \times \\
\times\left(\frac{L_{H A c k}}{B_{w d}}+t_{w d}\right)+\tau \times\left\{\begin{array}{l}
\max \left(\begin{array}{l}
\mathrm{H}_{C M R-N M R} \\
\mathrm{H}_{L M A-N M R}
\end{array}\right) \times \\
\left(\frac{L_{E P B U}}{B_{w d}}+t_{w d}\right)
\end{array}\right\}
\end{array}\right] \\
& \left.T_{P R}=\left[\begin{array}{l}
\frac{P_{w l f} \times \mathrm{H}_{S M R-N M R}}{1-P_{w l f}} \times \\
\times\left(\frac{L_{R S}}{B_{w l}}+t_{w l}\right)
\end{array}\right\}+\left(L_{\text {data }} \times t_{w l}\right)\right],
\end{aligned}
$$

where $P_{w l f}$ is the Wireless link failure probability, $L_{Z}$ is the Length of the $\mathrm{Z}$ message, $t_{w l}$ is the Wireless link delay and 
$t_{w d}$ Wired link delay. Correspondingly, the HD of NEMO BSP [19], [20] can be expressed as

$$
T_{H D}^{N E M O-B S P}=\mathrm{T}_{L S}+\mathrm{T}_{M D}+\mathrm{T}_{D A D}+\mathrm{T}_{S R}
$$

From (4), $T_{S R}$ is the Registration delay in NEMO BSP, $T_{D A D}$ is substituted as Retransmission Timer, Movement detection delay in NEMO BSP is defined by $T_{M D}$ [19], [20]. It is also assumed that the CoA is not exploited in any MNN at the access link. Accordingly, $T_{M D}$ and $T_{S R}$ can be expressed as:

$$
\begin{gathered}
\mathrm{T}_{M D}=\left[\begin{array}{c}
\frac{P_{w l f} \times \mathrm{H}_{M R-A R}}{1-P_{w l f}} \times\left\{\left(\frac{L_{R S}}{B_{w l}}+t_{w l}\right)+\right] \\
\left.+\left(\frac{L_{R A}}{B_{w l}}+t_{w l}\right)\right]
\end{array}\right], \\
\mathrm{T}_{S R}=\left[\begin{array}{c}
\frac{P_{w l f} \times \mathrm{H}_{M R-A R}}{1-P_{w l f}} \times\left\{\begin{array}{l}
\left(\frac{L_{B U}}{B_{w l}}+t_{w l}\right)+ \\
+\left(\frac{L_{B A}}{B_{w l}}+t_{w l}\right)
\end{array}\right) \\
{\left[\begin{array}{l}
\mathrm{H}_{A R-H A} \times\left(\frac{L_{B U}}{B_{w d}}+t_{w d}\right)+ \\
+ \\
+\mathrm{H}_{H A-A R} \times\left(\frac{L_{B A}}{B_{w d}}+t_{w d}\right)
\end{array}\right\},}
\end{array}\right],
\end{gathered}
$$

where $B_{w l}$ states the Bandwidth wireless link and $B_{w d}$ Bandwidth in the wired link. According to the PNEMO scheme [19], [6], the MAG2 directs a PBU message to LMA on behalf of MR for the handoff registration. It is not essential to send the PBU message via the wireless link since all the signaling is handled by the network side. Hence, HD of the PNEMO scheme is calculated as

$$
T_{H D}^{P N E M O}=\mathrm{T}_{L S}+\mathrm{T}_{R S}+\mathrm{T}_{L U}
$$

In (7), $\mathrm{T}_{R S}$ and $\mathrm{T}_{L U}$ is expressed as:

$$
\left.\begin{array}{c}
\mathrm{T}_{R S}=\frac{P_{w l f} \times H_{M R-M A G}}{1-P_{w l f}} \times\left(\frac{L_{R S}}{B_{w l}}+t_{w l}\right), \\
2 \times\left(\frac{H_{M A G-L M A} \times L_{P B U}}{B_{w d}}+t_{w d}\right)+\max \\
\mathrm{T}_{L U}=\left[\begin{array}{l}
2 \times\left(\frac{H_{M A G-L M A} \times L_{P B U}}{B_{w d}}+t_{w d}\right) \\
\mathrm{T}_{R S}^{P N E M O}+2 \times \tau \times \\
\times\left(\frac{H_{M A G-L M A} \times L_{P B U}}{B_{w d}}+t_{w d}\right)
\end{array}\right\}
\end{array}\right] .
$$

Therefore, the comparative handoff delay gain of the proposed multi-interfaced scheme with that of NEMO BSP, and PNEMO can be defined as follows:

$$
\begin{gathered}
G_{H D}=\frac{T_{H D}^{N E M O-B S P}}{T_{H D}^{M \text { Multi-int erfaced }},} \\
G_{H D}=\frac{T_{H D}^{P-N E M O}}{T_{H D}^{M u l t i-\text { int } \text { erfaced }},} \\
G_{H D}=\frac{T_{H D}^{N E M O-B S P}}{T_{H D}^{P N E M O}} .
\end{gathered}
$$

The multi-interfaced SMR is capable to support flowbased fast registration process in the proposed scheme. Thus, it is possible to prevent the Packet Loss (PL) during handoff as mentioned in (1). According to the proposed scheme, the LMA begins to forward data packets to the NMR once it receives the EPBU message from NMR via the wired link. Since the number of packet loss is proportionate to the total handoff delay, hence, the total packet loss for the proposed scheme can be obtained by

$$
\begin{gathered}
T_{P L}^{\text {Multi-interfaced }}=\lambda_{s} \times \mu_{h} \times N_{S M R} \times \\
\times\left[T_{L S}+\mathrm{H}_{N M R-C M R} \times\left(\left(\frac{L_{H I}}{B_{w d}}+t_{w d}\right)+\left(\frac{L_{H A c k}}{B_{w d}}+t_{w d}\right)\right)\right] .
\end{gathered}
$$

In (13), average session length and mobility rate are symbolized as $\lambda_{s}$ and $\mu_{h}$. In addition to that, the Number of SMR $\left(N_{S M R}\right)$ plays an important role. This is because; the packet loss is directly equivalent to the rate of handoffs it is exposed within a particular time.

In the case of the NEMO BSP and PNEMO, the MR is not capable to get any packets from its HA or LMA at the time handoff till it finishes location update registration procedure with the HA or LMA. Hence, packet loss for NEMO BSP and PNEMO can be expressed as:

$$
\begin{gathered}
T_{P L}^{N E M O-B S P}=\lambda_{s} \times \mu_{h} \times N_{M R} \times\left(T_{H D}^{N E M O-B S P}\right), \\
T_{P L}^{P N E M O}=\lambda_{S} \times \mu_{h} \times N_{M R} \times\left(T_{H D}^{P N E M O}\right) .
\end{gathered}
$$

\section{B. Simulation Evaluation in Ns 3}

This sub-section evaluates the performance of the proposed multi-interfaced fast registration mechanism via NS-3 Network Simulator. The NS-3 is a discrete-event network simulator for Internet systems [20]. It has a good development momentum and provides a better core architecture. It should be especially noted that NS-3 fully supports multiple interfaces. NetAnim and gnuplot are used in order to analyse, visualize or process the data gained through simulation. The cycle of simulation steps using NS3 simulator is depicted in Fig. 3.

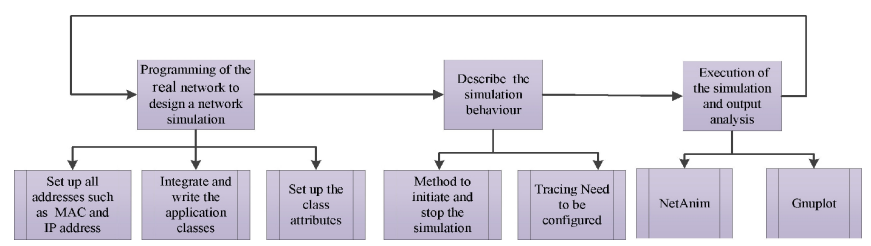

Fig. 3. Simulation steps using NS3 Simulator. 
The considered simulation scenario is shown in Fig. 4 in which PMIPv6 is used with WiFi and $3 \mathrm{G}$ interfaces network environment.

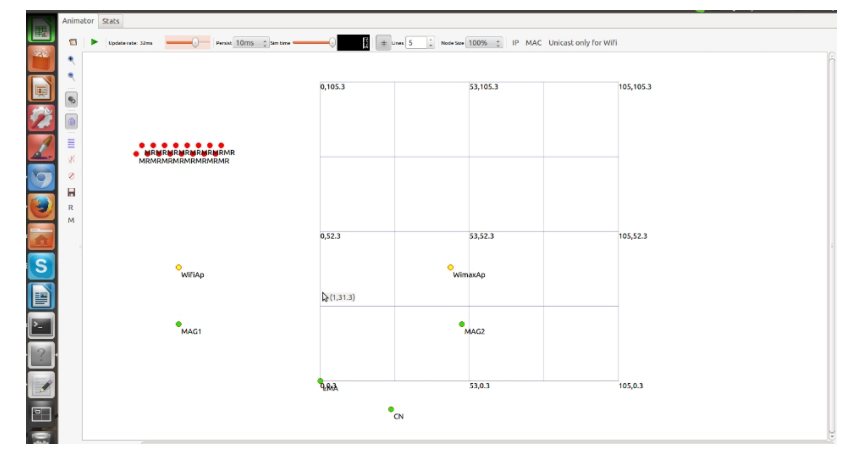

Fig. 4. Simulation scenario for the proposed scheme.

Initially, the SMR is connected to the WiFi network while the $3 \mathrm{G}$ is idled. The performance of the proposed scheme is compared with that of PNEMO scheme since it has a better performance in comparison to the standard NEMO-BSP.

\section{RESULTS ANALYSIS}

\section{A. Numerical Results}

This subsection illustrates the comparative numerical results of the proposed multi-interfaced scheme. It quantitatively compares the performance of proposed scheme with NEMO BSP and PNEMO with regard to the handoff delay as well as packet loss. The numerical results are achieved via implementing the developed analytical modeling in MATLAB (R2013b) by using the parameter values as listed in Table I [19], [20].

TABLE I. PARAMETERS FOR ANALYTICAL ANALYSIS.

\begin{tabular}{|c|c|}
\hline Parameter & Value \\
\hline$N_{S M R}$ & $1-20$ \\
\hline$T_{L S}$ & {$[50-300] \mathrm{msec}$.} \\
\hline$T_{D A D}$ & $1000 \mathrm{millisecond}$ \\
\hline$B_{w l}$ & $54 \mathrm{Mb} / \mathrm{s}$ \\
\hline$B_{w d}$ & $1000 \mathrm{Mb} / \mathrm{s}$ \\
\hline$t_{w l}$ & 2 millisecond \\
\hline$t_{w d}$ & 0.5 millisecond \\
\hline$H_{M R-A R}=H_{M R-M A G}$ & 1 \\
\hline$H_{\text {FLMR-FMR }}=H_{\text {FMR }}=H_{H A-A R}=H_{L M A-M A G}$ & 5 \\
\hline$H_{C F M R-N F M R}=H_{A R-A R}=H_{M A G I-M A G 2}$ & 1 \\
\hline$L_{E P B A}=L_{B A}=L_{P B A}=L_{R A}$ & 52 Bytes \\
\hline$L_{E P B U}=L_{B U}=L_{P B U}$ & 72 Bytes \\
\hline$L_{H I}=L_{H A c k}$ & 52 Bytes \\
\hline$L_{R A}$ & 92 Bytes \\
\hline$\lambda_{S}$ & {$[10-35]$ packets/flow } \\
\hline$E\left(T_{S M R}\right)$ & {$[10-100]$ second } \\
\hline
\end{tabular}

The influence of link switching delay (TLS) on handoff delay gain is illustrated in Fig. 5. Usually, TLS is dependent on the wireless access technology. From Fig. 5, it is shown that, as TLS decreases, the comparative handoff delay gain increases for all schemes. However, it is also noticed from plotted Figure that, the overall gain of PNEMO decreases as $\tau$ increases, whereas the multi-interfaced scheme remains almost the same, regardless of the increasing $\tau$. This is due to the tunnelling burden of PNEMO that effects in disruption of session continuity. $\tau$ indicates the amount of traffic density over the link among the access routers in the proposed scheme. When road traffic is high, the number of SMRs moving between the same interfaces increase. Therefore, $\tau$ can imply road traffic characteristics. Since, the delay over wireless access link rises, the handoff delay rises in multiinterfaced scheme. However, these delays are not critical as in NEMO-BSP. From the observation in Fig. 14, it is confirmed that the proposed multi-interfaced scheme and PNEMO scheme show frequent handoff compared to NEMO-BSP. This is because, the location update over the wireless link is avoided and no tunnelled packets are conveyed over the wireless link. Moreover, the DAD mechanism in NEMO-BSP counts for a huge portion of handoff delay. Hence, it is essential to reduce this DAD delay to improve handoff performance. Subsequently, multiinterfaced scheme also outperforms PNEMO by taking the advantage of previous knowledge about the network conditions and its flows during flow-enabled fast registration phase. Therefore, it can be summed up from Fig. 15 that, the multi-interfaced scheme can vastly improve the handoff performance compared to NEMO BSP and PNEMO regardless of increasing $T_{L S}$ and $\tau$. This is because, SMR is capable to split the application flow burden among multiple access technology during inter-technology handoff.

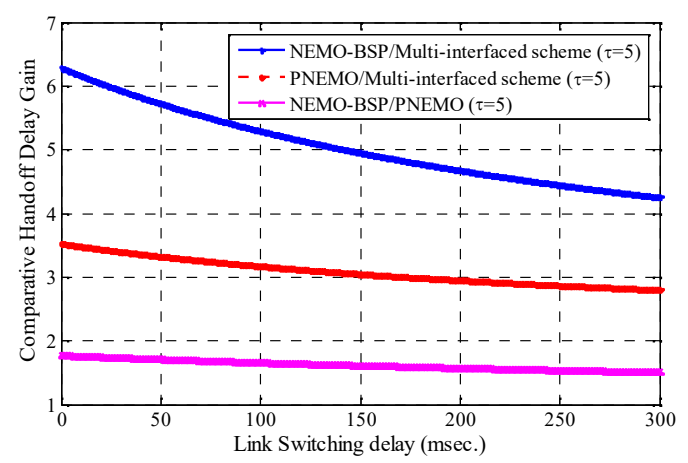

Fig. 5. Handoff delay gain vs. Link Switching Delay.

In Fig. 6, the amount of packet loss during inters technology handoff for each scheme is depicted for varying the cell residence time $\left(T_{S M R}\right)$ with different number of SMRs. If the $T_{S M R}$ is varying from $20 \mathrm{sec}$ to $100 \mathrm{sec}$, the SMR is most likely to stay in a cell and rarely moves to another position. Thus, the packet loss during handoff is very small. When mobility rate increases with the number of SMRs, the SMR moves frequently and changes subnet recurrently because of its high mobility.

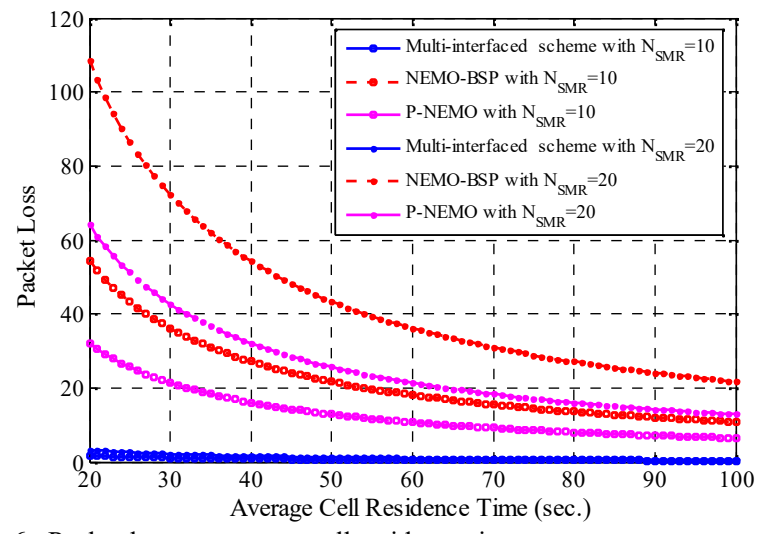

Fig. 6. Packet loss vs. average cell residence time. 
Thus, includes a number of SMR handoffs which leads to increase packet loss as depicts in Fig. 6. Basically, packet loss is proportional to the handoff disruption time. Therefore, the cases of the handoff mode for multiinterfaced scheme shows lower packet loss compared to PNEMO and NEMO BSP.

\section{B. Simulation Results}

The parameters are detailed in Table II [19].

TABLE II. PARAMETERS USED FOR SIMULATION.

\begin{tabular}{|c|c|}
\hline Parameter & Value \\
\hline Simulator & NS3.12.1 \\
\hline Operating system & Linux Ubuntu 12.04 \\
\hline Simulation time & {$[0-10]$ sec. } \\
\hline Packet size & 1024 bytes \\
\hline SMR speed & {$[30,40]$ sec. } \\
\hline No. of connections per SMR & 2 \\
\hline Types of technologies supported by SMR & WiFi and 3G \\
\hline Pause time & 12 millisecond \\
\hline Mobility model & $\begin{array}{c}\text { Constant velocity mobility } \\
\text { model. }\end{array}$ \\
\hline
\end{tabular}

The time discrepancy among the moment of notification of the approaching handoff at the previous link as well as the moment the SMR directly gets the first data packets on the new link can be denoted as the handoff delay. In case of real-time application, this handoff delay is a crucial performance metrics. The variations of average handoff delay during inter-technology handoff for the proposed scheme and PNEMO scheme is analysed in Fig. 7 and Fig. 8 respectively with changing the time.

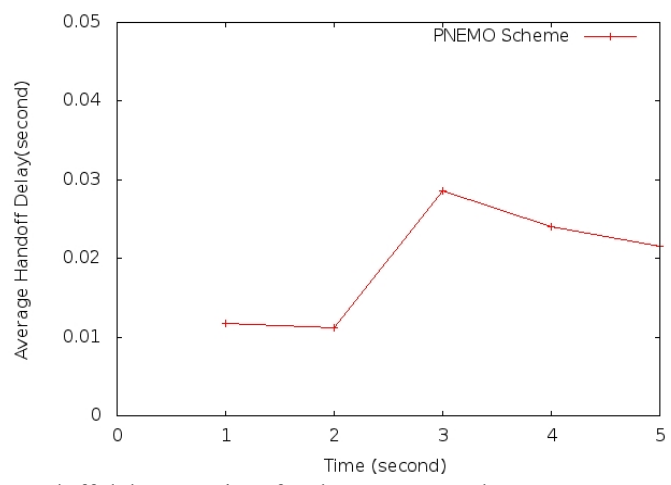

Fig. 7. Handoff delay vs. Time for the PNEMO scheme.

Initially, the SMR passes from the network coverage area of Physical Interface-1 (PI-1) to Physical Interface-2 (PI-2). Analysing Fig. 7 for speed $=30 \mathrm{~m} / \mathrm{sec}$ and the number of SMR is 10 , it is noticed that the handoff delays of the PNEMO scheme escalate linearly with raising the time from 1 second to 3 second. This result is related to the number of handoffs occurred and the network status at the time of the handoff. The average handoff delay experienced by the PNEMO scheme is .021 second as plotted in Fig. 7. Likewise, Fig. 8 illustrates the variation of the average handoff delay experienced by the proposed scheme during inter-technology handoff as the SMR performs the handoff from CMR to NMR. The average handoff delay is .011 seconds for the number of SMR is 10 and the speed is $40 \mathrm{~m} / \mathrm{second}$ as depicts in Fig. 8. The number of handoffs is significantly reduced in the proposed scheme due to the use of multi-interfaced fast handoff technique during intertechnology handoff as appeared in Fig. 8.

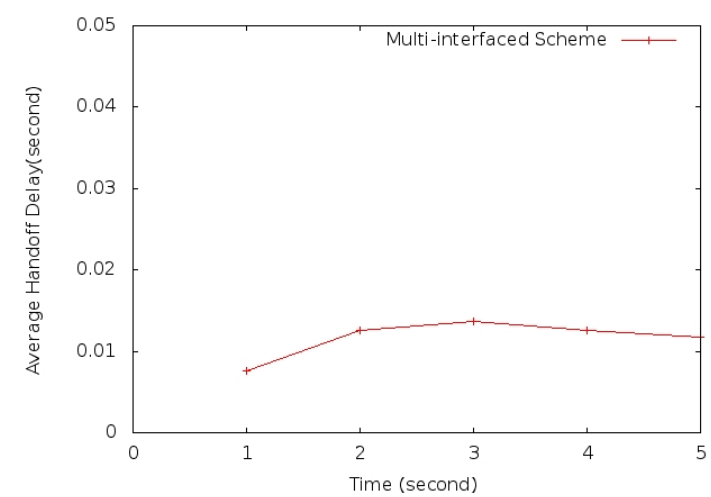

Fig. 8. Handoff delay vs. Time for the proposed scheme.

The is due to the fact that, the moment handoff is predicted via L2 triggering, signaling messages for fast registration are exchanged between $\mathrm{CMR}$ and NMR in the proposed scheme. Therefore, the handoff procedure is accomplished within much smaller time. Thus, the proposed scheme can evade unnecessary modifications compared to PNEMO scheme.

Packet loss can be stated as the failure or distortion of some conveyed data packets to arrive at the destined receiver. This incident is capable to cause an obvious effect in each kind of time-sensitive applications in real world. As there is a link between handoff delay and its impact on packet loss, henceforth the variation of the packet loss for the proposed scheme and PNEMO scheme is illustrated in Fig. 9.

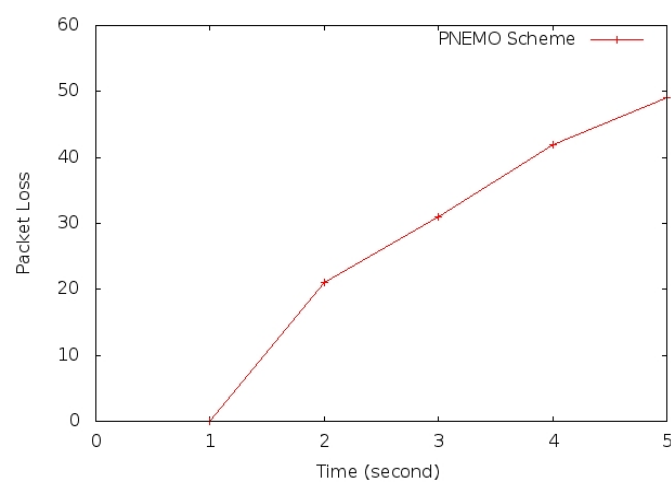

Fig. 9. Packet loss vs. Time for the PNEMO scheme.

Figure 10 shows the packet loss over the time.

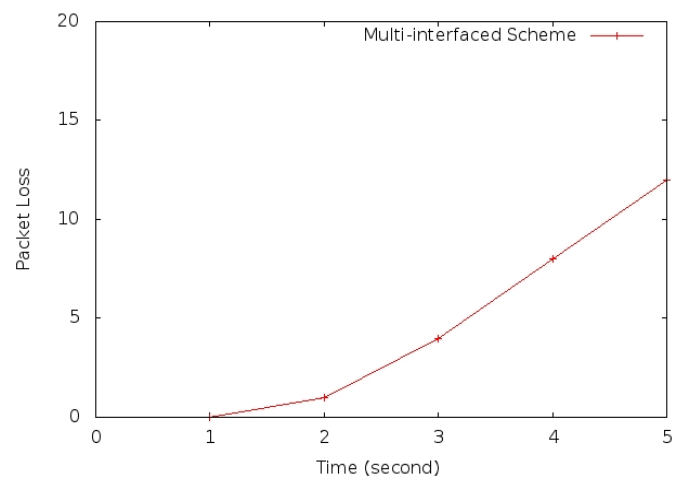

Fig. 10. Packet loss vs. time for the proposed scheme. 
The time is varied from 1 second to 5 second; whereas the number of SMR is kept constant (i.e. SMR = 10). It is indicated from the same plotted figures that, the packet loss variation occurs according to the change of time, once the SMR travels between PIs to support inter-technology handoff. From both plotted figures, it is noticeable that, applying multiple interfaces in P-NEMO environment has a supportive influence on the packet loss for the proposed scheme.

\section{CONCLUSIONS}

A multi-interfaced fast handoff scheme is proposed to support continuous Internet connectivity in PNEMO environment. The performance of the proposed scheme is evaluated via numerical and simulation approaches. In order to implement an extensive simulation scenario as well as the modeling of the proposed scheme, the new code is integrated with the current modules in NS-3 simulation environment. The proposed scheme has been compared with the PNEMO and NEMO BSP scheme for benchmarking. The extensive analysis of handoff delay is discussed considering the WiFI and $3 \mathrm{G}$ interface. It is observed that the multi-interfaced fast handoff is more suitable and robust in term of cell residence delay, and lowered handoff delay. It has been also observed that the proposed scheme has achieved significant improvement in reducing the packet loss comparing to the single interface-based PNEMO scheme and NEMO BSP. The experimental test bed is considered as future work for more accurate evaluation on the end-to-end delay of the proposed scheme. Moreover, cell residence and handoff delay is to be analyzed in the $5 \mathrm{G}$ multi accessed network.

\section{REFERENCES}

[1] K. Zhu, D. Niyato, P. Wang, E. Hossain, D. Kim, "Mobility and handoff management in vehicular networks: a survey", Wireless communications and mobile computing, vol. 11, no. 4, pp. 459-476, 2011. DOI: $10.1002 / \mathrm{wcm} .853$.

[2] I. Al-Surmi, M.Othman, B. M. Ali, "Mobility management for IPbased next-generation mobile networks: Review, challenge, and perspective", Journal of Network and Computer Applications, vol. 35, no. 1, pp. 295-315, 2012. DOI: 10.1016/j.jnca.2011.09.001.

[3] B. Chandavarkar, G. R. M. Reddy, "Survey paper: Mobility management in heterogeneous wireless networks", Procedia Engineering, vol. 30, pp. 113-123, 2012. DOI: 10.1016/j.proeng.2012.01.841.

[4] M. Zekri, B. Jouaber, D. Zeghlache, "A review on mobility management and vertical handover solutions over heterogeneous wireless networks", Computer Communications, vol. 35, no. 17, pp. 2055-2068, 2012. DOI: 10.1016/j.comcom.2012.07.011.
[5] V. Devarapalli, R. Wakikawa, A. Petrescu, P. Thubert, Network mobility (NEMO) basic support protocol, RFC 3963, 2004.

[6] R. Wakikawa, V. Devarapalli, G. Tsirtsis, T. Ernst, K. Nagami, Multiple care-of addresses registration, RFC 5648, 2009.

[7] M. Calderon, C. Bernardos, I. Soto, PMIPv6 and Network Mobility Problem Statement. 2012.

[8] S. Ryu, C. Ji-Woong, P. Kyung-Joon, "Performance evaluation of improved fast PMIPv6-based network mobility for intelligent transportation systems", Journal of Communications and Networks, vol. 15, no. 2, pp. 142-152, 2013. DOI: 10.1109/JCN.2013.000027.

[9] R. Kuntz, J. Montavont, G. Schreiner, D. Binet, T. Noel, "An improved network mobility service for intelligent transportation systems", Wireless communications and mobile computing, vol. 1, no. 7, pp. 899-915, 2011. DOI: $10.1002 / \mathrm{wcm} .860$.

[10] C.-W. Lee, M. C. Chen, Y. S. Sun, "A novel network mobility management scheme supporting seamless handover for high-speed trains", Computer Communications, vol. 37, pp. 53-63, 2014. DOI: 10.1016/j.comcom.2013.09.009.

[11] J.-H. Lee, T. Ernst, "Lightweight network mobility within PMIPv6 for transportation systems", IEEE Systems Journal, vol. 5, no. 3, pp. 352-361, 2011. DOI: 10.1109/JSYST.2011.2158681.

[12] J.-H. Lee, T. Ernst, N. Chilamkurti, "Performance analysis of PMIPv6-based network mobility for intelligent transportation systems", IEEE Trans. Vehicular Technology, vol. 61, no. 1, pp. 7485, 2012. DOI: 10.1109/TVT.2011.2157949.

[13] S. Ryu, J.-W. Choi, K.-J. Park, "Performance evaluation of improved fast PMIPv6-based network mobility for intelligent transportation systems", Journal of Communications and Networks, vol. 15, no. 2, pp. 142-152, 2013. DOI: 10.1109/JCN.2013.000027.

[14] S. Krishnan, H. Yokota, T. Melia, C. Bernardos, Issues with network based inter-technology handovers. IETF draftkrishnannetextintertech-ps-02, 2009.

[15] X. Chen, H. Zhang, Y.-C. Chang, H.-C. Chao, "Experimentation and performance analysis of multi-interfaced mobile router scheme", Simulation Modelling Practice and Theory, vol. 18, no. 4, pp. 407415, 2012. DOI: 10.1016/j.simpat.2009.09.005.

[16] H.-D. Park, K.-N. Park, A Multihoming-Based Vertical Handover Scheme IT Convergence and Security. Springer, 2013, pp. 749-754. DOI: $10.1007 / 978-94-007-5860-589$.

[17] R. Kuntz, J. Montavont, T. Noel, "Multihoming in IPv6 mobile networks: progress, challenges, and solutions", IEEE Communications Magazine, vol. 51, no. 1, pp. 128-135, 2013. DOI: 10.1109/MCOM.2013.6400449.

[18] M. S. Hossain, M. Atiquzzaman, W. Ivancic, "Performance evaluation of multihomed NEMO", IEEE Int. Conf. Communications (ICC 2012), Ottawa, ON, Canada, 2012, pp. 5429-5433. DOI: 10.1109/ICC.2012.6364465

[19] J. H. Lee, T. Ernst, "Lightweight network mobility within PMIPv6 for transportation systems", IEEE Systems Journal, vol. 5, no. 3, pp. 352-361, 2011. DOI: 10.1109/JSYST.2011.2158681.

[20] J. H. Lee, T. Ernst, N. Chilamkurti, "Performance analysis of PMIPv6-based network mobility for intelligent transportation systems", IEEE Trans. Vehicular Technology, vol. 61, no. 1, pp. 7485, 2012. DOI: 10.1109/TVT.2011.2157949.

[21] H.-Y. Choi, S.-G. Min, Y. H. Han, J. Park, H. Kim, "Implementation and evaluation of proxy mobile IPv6 in NS-3 network simulator", in Proc. 5th Int. Conf. the Ubiquitous Information Technologies and Applications (CUTE 2010), Sanya, China, 2010, pp. 1-6. DOI: 10.1109/ICUT.2010.5677817. 